\title{
Control Emocional, Preocupación por la Covid-19 y Comportamiento del Consumidor en la Población Limeña
}

\author{
Emotional Control, Concern about Covid-19 and Consumer Behaviour in \\ the Population of Lima
}

\author{
Manuel Torres Lajo ${ }^{1}$ \\ Technische Universität München
}

Manuel Torres Valladares², Oscar Tinoco Gómez ${ }^{3}$

Universidad Nacional Mayor de San Marcos

Recibido: $19-03-21$

Aceptado: 29 - $11-21$

Publicado: $23-12-21$

\begin{abstract}
Resumen
Esta investigación tiene como objetivo establecer si el control emocional, la preocupación por la COVID-19 y el comportamiento del consumidor se encuentran vinculados entre sí; así como establecer las actitudes de la población de Lima, Perú, frente a la pandemia y sus hábitos de consumo. Se elaboraron tres cuestionarios bajo un formato digital, que fueron resueltos por 663 sujetos de Lima. Los resultados muestran que existe una relación significativa entre control emocional y la preocupación por la COVID-19, pero no así entre dicha preocupación y el comportamiento del consumidor. En cambio, los resultados descriptivos sí muestran cambios en el comportamiento del consumidor como resultado de la pandemia. Estos resultados permiten concluir que, a pesar de que la COVID-19 sigue siendo una preocupación para los pobladores, es muy probable que se haya controlado el temor inicial, sea por adaptación a la nueva normalidad, o por la necesidad imperiosa de salir a trabajar para sobrevivir.
\end{abstract}

Palabras clave: COVID-19; Control Emocional; Comportamiento del Consumidor; Hábitos de Consumo.

JEL: D12, D91, E71

1 Economista, Master en Ciencias por la Technische Universität München, Alemania

Autor para correspondencia: mptorreslajo@me.com ORCID: https://orcid.org/0000-0003-3085-1394

2 Docente Principal de la Facultad de Psicología - Universidad Nacional Mayor de San Marcos, Lima, Perú.

E-mail: mtorresv@unmsm.edu.pe ORCID: https://orcid.org/0000-0003-1530-4229

3 Docente Principal Facultad Ingeniería Industrial - Universidad Nacional Mayor de San Marcos, Lima, Perú. E-mail: otinocog@gmail.com ORCID: https://orcid.org/0000-0002-7927-931X

(C) Los autores. Este artículo es publicado por la Revista de Investigación en Psicología de la Facultad de Psicología, Universidad Nacional Mayor de San Marcos. Este es un artículo de acceso abierto, distribuido bajo los términos de la licencia Creative Commons Atribución 4.0 Internacional (CC BY 4.0) [https://creativecommons.org/licenses/by/4.0/deed.es] que permite el uso, distribución y reproducción en cualquier medio, siempre que la obra original sea debidamente citada de su fuente original. 


\begin{abstract}
This research's objective is to establish whether the emotional control, the concern about COVID-19 and the consumer behaviour are related among them; as well as to establish the attitudes of the population from Lima, Peru towards the pandemic and its consumption habits. Three digital questionnaires were made, which were answered by 663 people from Lima. The results show that a significant relationship exists between emotional control and the concern about COVID-19; but there is not one between such concern and the consumer behaviour. On the other side, the descriptive results do show changes in the consumer behaviour as a result of the pandemic. These results lead to the conclusion that, even though COVID-19 is still a concern for the population, it is highly likely that the initial fear has been controlled either due to adaptation to the new normality or due to the imperious necessity to go out to work to survive.
\end{abstract}

Keywords: COVID-19; Emotional Control; Consumer Behaviour; Consumption Habits.

JEL: D12, D91, E71 
La pandemia de la COVID-19, declarada oficialmente así por la Organización Mundial de la Salud (OMS) en marzo de 2020, trajo consigo grandes y rápidos cambios en los estilos de vida de las personas a nivel mundial, pues, de un momento a otro, la vida en sociedad dejó de ser la que conocíamos. Así, diversos gobiernos decretaron cuarentenas y cierres de actividades no esenciales - entre ellos el Perú el 15 de marzo de 2020 (Presidencia del Consejo de Ministros, 2020)—, con el objetivo de frenar la expansión del virus, forzando a buena parte de la población a quedarse en casa. El miedo a la incertidumbre y al contagio del virus, entre otros, hicieron que se formaran grandes filas frente a los comercios (Garbe, Rau \& Toppe, 2020), donde se compraba compulsivamente papel higiénico, productos no perecibles - como pastas o alimentos en conserva-, así como de alcohol en gel y otros productos de limpieza. No fue un fenómeno exclusivamente peruano (Radio Programas del Perú, 2020) ni de otros países latinoamericanos como Chile (Díaz, 2020), pues se vio también en países desarrollados como Australia, Alemania, España y Estados Unidos (Grace \& Sakkal, 2020; Stalinski, 2020; Bécares, 2020; Corkery \& Maheshwari, 2020).

Estas reacciones - miedo, fatiga, pánico y otros problemas asociados- ante lo desconocido y/o sobre lo que no se tiene control ni se puede anticipar su desenlace son "normales" (Taylor, 2019), particularmente en eventos disruptivos como este. En corto tiempo, la población debió habituarse a una "nueva normalidad", en la que el distanciamiento social, las medidas de higiene personal reforzadas, el uso de mascarillas, las restricciones de movilidad, entre otros, se hicieron cotidianos. De su cumplimiento exitoso dependía el evitar una propagación más descontrolada. Pero no era una tarea sencilla, ya que esas restricciones podían desencadenar las reacciones emocionales mencionadas anteriormente, con consiguientes incrementos en los casos de estrés, ansiedad y angustia.

Es en este contexto que se pone en ejercicio el manejo y control de las emociones. Esto se puede entender como una competencia que le permite al ser humano enfrentar y resolver adecuadamente las diferentes situaciones emocionales, desde las más simples a las más estresantes, sin perder la ecuanimidad y efectividad (Goleman, 2015). La analogía del sistema dual del cerebro humano, presentada por psicólogos como Kahneman (2011), permite entender por qué los seres humanos ven el mundo desde una perspectiva diferente cuando las emociones toman el control (Ariely, 2010). Bajo el sistema dual se tiene que, el Sistema 1 actúa emocionalmente y de forma automática, y el Sistema 2, de forma racional y meditada (Kahneman, 2011). El Sistema 2 es lento para reaccionar, y en situaciones de alto estrés, como el estado de alerta en el que se ha vivido en los últimos meses, las decisiones tienden a ser emocionales, pues el Sistema 1 es rápido para decidir y se impone al Sistema 2. En términos de Mullainathan y Shafir (2014), cuando el cerebro ha alcanzado su capacidad máxima de "ancho de banda" dada una preocupación (o una escasez), el ser humano tiene una menor capacidad para 
atender otros asuntos, por lo que actúa de forma impulsiva y su capacidad para ejercer control sobre sus emociones es menor.

Ante una situación potencialmente estresante que podía devenir en problemas emocionales, el gobierno peruano optó, al inicio de la emergencia, por realizar mensajes comunicacionales muy directos con la población, sea a través de campañas o conferencias de prensa del Presidente de la República con una periodicidad diaria. El objetivo detrás de esto no era otro que dar a conocer, de primera mano, la situación epidemiológica del país y comunicar directamente las nuevas medidas de restricción adoptadas. Así, se buscaba dar tranquilidad y seguridad a la población. A pesar de estos esfuerzos, la curva de contagios fue aumentando progresivamente (Worldometer, 2020) y con el pasar de los meses, una parte de la población, ante la incertidumbre de una crisis ya no solo sanitaria sino económica, comenzó a ignorar las medidas y propugnar la idea de cada persona debe cuidar de sí misma —idea eventualmente secundada por el presidente (TVPerú, 2020) - y de retomar sus actividades económicas habituales (Agencia EFE, 2020). Esto se debe, entre otros, a la alta informalidad de la economía peruana: un $74.3 \%$ de los peruanos trabaja en el sector informal, según cifras del INEI citadas por la Organización Internacional del Trabajo (Gamero y Pérez, 2020). La informalidad trae consigo un flujo de ingresos no constante y precariedad laboral, lo que obliga a los trabajadores a trabajar día a día para generar ingresos y/o no perder su trabajo.

Todo ello pese a la implementación por parte del gobierno de programas de ayuda económica, como bonos, a diversos grupos vulnerables (Salazar, 2020). No obstante, los problemas en su entrega, el monto (760 soles, un equivalente aproximado a 210 USD), y el tiempo que había transcurrido desde el inicio de la emergencia cuando se paralizaron las actividades no esenciales, hicieron que la población opte por salir a trabajar, provistos de mascarillas, alcohol en gel y otros equipos de protección personal. El miedo al contagio y a la crisis económica son elementos de presión que pueden devenir en un incremento de cuadros de estrés $\mathrm{y}$ angustia.

Tal como se ha mencionado anteriormente, las restricciones derivadas de la pandemia de la COVID-19 han impactado en la economía de la población, lo que tiene una expresión inmediata en su capacidad de consumo. El comportamiento del consumidor ha tenido un cambio forzoso: se pasó de compras presenciales a compras online, de compras pequeñas cada día a compras más grandes en una sola visita, se priorizó la compra de productos de primera necesidad, entre otros.

En este panorama es que se desarrolla la presente investigación, en la que se pretende examinar el papel del control emocional frente a la preocupación por la pandemia de la COVID-19 y cómo esto puede haber alterado los hábitos de consumo de los pobladores de la ciudad de Lima. Se entiende que las emociones son estados psicológicos muy importantes para explicar el comportamiento, la toma 
de decisiones, la regulación cognitiva y las competencias de interacción social entre los seres humanos (Gross, 2009). Por estas razones es que se considera que la emoción tiene influencia directa sobre toda la experiencia humana, en tanto ordena y regula el comportamiento humano y lo puede direccionar hacia el logro de sus objetivos (Grolnick, McMenamy \& Kurowski, 2006).

Es verdad que las experiencias emocionales no pueden ser independientes de la cultura y, precisamente, es eso lo que permite su control, regulación y evaluación (De Leersnyder, Boiger \& Mesquita, 2013), necesarias para poder dirigir correctamente la conducta frente a cualquier situación que se pueda presentar como es, por ejemplo, el caso de la pandemia que vive el mundo. De acuerdo con lo señalado por Del Pozo (2013), el control y regulación emocional le permiten al sujeto manejar las emociones propias, pero también la de los demás, sobre todo cuando se enfrentan a situaciones muy intensas, de lo contrario, las respuestas emocionales se pueden desbordar y en esa situación se pueden generar comportamientos inadecuados que afecten el cumplimiento de sus actividades regulares, y hasta su calidad de vida (Chóliz, 2005; Piqueras et al., 2009).

Los problemas referidos al control emocional (De Leersnyder, et al., 2013; Del Pozo, 2013; Chóliz, 2005; Piqueras et al., 2009), podría explicar las compras nerviosas que se produjeron al inicio de la pandemia y en la que las personas trataron de abastecerse de un conjunto de productos de primera necesidad, desbordando la capacidad de atención de los supermercados (Garbe, et al., 2020). De alguna manera se trataba de tener una suerte de control emocional frente a una situación caracterizada por la incertidumbre, miedo y hasta pánico, al no tener claridad sobre la naturaleza de la enfermedad, más allá de que podría conducir a la muerte.

Progresivamente las personas fueron adaptándose a la nueva situación, ya sea porque obtuvieron un mayor conocimiento sobre la enfermedad y como controlarla, o porque requerían salir a trabajar para poder obtener el dinero que les permita sobrevivir. Es claro que las recomendaciones de quedarse en casa y aprovechar el tiempo para reflexionar, hacer ejercicios, practicar yoga, leer libros o aprender repostería, etc., no funcionan en personas que requieren trabajar día a día para poder satisfacer sus necesidades básicas como alimentarse. Se presenta así, una disyuntiva — salud versus economía — que el gobierno no ha podido controlar.

A finales del 2020, nueve después del inicio del estado de emergencia en el Perú, la situación nos mostraba un retorno prácticamente total a la situación prepandemia — aún cuando algunas actividades continuaban restringidas-, solo que con mascarillas y alcohol: el tránsito era nuevamente caótico, se formaban largas colas en los accesos a los centros y calles comerciales, etc. Pese a ello, las estimaciones de la recuperación del consumo (Salas, 2020) nos muestran que este sigue orientado al consumo de productos de primera necesidad, lo que implica una contracción en los gastos de productos no esenciales. 
Las preguntas de investigación que se propusieron en la presente investigación estuvieron orientadas a resolver cuestiones como: ¿Qué relaciones existen entre el control emocional, la preocupación por la COVID-19 y el comportamiento del consumidor en la población limeña? ¿Cuál es el comportamiento de las variables control emocional, preocupación por la COVID-19 y comportamiento del consumidor en la población limeña?

\section{MÉTODO}

La presente investigación, de enfoque cuantitativo y con un diseño no experimental de nivel correlacional, se realizó del primero al quince de setiembre del 2020. En aquel momento, el Perú promediaba entre 6000 y 7000 casos nuevos al día (Worldometer, 2020) y, al 10 de setiembre, se ubicaba en el quinto lugar en el mundo en casos confirmados (La Nación, 2020).

\section{Muestra}

Se trabajó con una muestra de 663 adultos de ambos sexos residentes en los diferentes distritos de la ciudad de Lima, considerando su tamaño poblacional. La participación de los sujetos de la muestra fue totalmente anónima y con su libre consentimiento. El instrumento fue enviado por correo electrónico utilizando un formulario digital y las respuestas se recibieron de la misma manera. Del total de encuestados, un $64.3 \%$ son mujeres, y la mayoría proceden de distritos pertenecientes a Lima Norte (25.5\%) y Lima Este (25\%) (para mayor detalle ver tabla 1).

Tabla 1

Distribución de la muestra

\begin{tabular}{lcc}
\hline Muestra & Media & \% \\
\hline Sexo & & \\
\cline { 1 - 1 } Masculino & 237 & 35.7 \\
Femenino & 426 & 64.3 \\
\hline Sector (distritos) & & \\
\cline { 1 - 1 } Lima Norte & 169 & 25.5 \\
Lima Centro & 50 & 7.5 \\
Lima Moderna & 84 & 12.7 \\
Lima Este & 166 & 25.0 \\
Lima Sur & 127 & 19.2 \\
Callao & 67 & 10.1 \\
\hline
\end{tabular}

\section{Instrumentos}

Se utilizó tres instrumentos que fueron construidos para la ocasión, cumpliendo todas las exigencias técnicas que se reclaman en estos casos. Los ítems fueron 
sometidos al proceso de criterio de jueces, para lo cual se solicitó el apoyo de ocho especialistas con la experiencia necesaria para verificar que los ítems estén claramente redactados y si representan lo que se pretende evaluar. A partir de la calificación obtenida a través del criterio de jueces, se estimó el coeficiente de $\mathrm{V}$ de Aiken (Escurra, 1988) en el que se tuvo en cuenta que, al participar ocho jueces, por lo menos siete de ellos debían dar opinión favorable, lo que en efecto ocurrió. Posteriormente se procedió a realizar los ajustes necesarios y someter los instrumentos a los análisis de confiabilidad respectivos. Finalmente, la prueba quedo conformada por 10 ítems para la escala de control emocional, (alfa de Cronbach de $0,80)$ y 11 ítems para el comportamiento del consumidor (Kuder Richardson $20=$ 0,83). Para la preocupación por la COVID-19, se tomó el ítem (“¿Qué tan grande es su preocupación respecto al coronavirus?"), formulado por Garbo, et al., (2020), la misma que se debía responder considerando cinco alternativas según una escala tipo Likert: Muy grande (1), Grande (2), Regular (3), Poco (4), Muy poco (5). Este ítem también obtuvo la opinión favorable de los jueces consultados.

\section{RESULTADOS}

Los resultados expuestos en la tabla 2, indican que, en efecto, los sujetos de la muestra se sienten amenazados por la pandemia de la COVID-19: un 57.7\% afirma tener una preocupación muy grande $(26.2 \%)$ o grande (31.5\%). Estos resultados son la expresión de la población frente a una situación que les es desconocida, sin forma de vencerla a corto plazo, que les genera pérdidas laborales y económicas, que limita su vida y la oportunidad de ganar dinero y mantener a sus familias o simplemente de no poder salir y hacer una vida normal.

\section{Tabla 2}

Preocupación por la COVID-19: ¿Qué tan grande es su preocupación respecto al coronavirus?

\begin{tabular}{lc}
\hline Preocupación & \% \\
\hline Muy grande & 26.2 \\
Grande & 31.5 \\
Regular & 23.7 \\
Poco & 11.9 \\
Muy poco & 6.6 \\
Total & 100.0 \\
\hline
\end{tabular}

Los resultados que se presentan en la Tabla 3 dan cuenta de una diversidad de respuestas que se ubican entre el término medio (Ni de acuerdo ni en Desacuerdo) y en las respuestas más positivas, que podrían hacer pensar que podrían enfrentar situaciones difíciles como las de la pandemia, sin embargo, el miedo existió y las compras nerviosas también, aunque es verdad, también, que estas compras 
se aquietaron con alguna rapidez y los problemas de escasez fueron rápidamente controlados. Es importante también resaltar la gran capacidad de resiliencia que tiene la población peruana que les ha permitido resistir una importante cantidad de desastres naturales, pandemias y la pobreza que es un problema permanente y con la que se lucha a diario.

Tabla 3

Control emocional

\begin{tabular}{lcccccc}
\hline Respuestas & & $\mathbf{1}$ & $\mathbf{2}$ & $\mathbf{3}$ & $\mathbf{4}$ & $\mathbf{5}$ \\
\hline Soy bueno para resistir las tentaciones & $\mathrm{f}$ & 12 & 68 & 189 & 267 & 127 \\
& $\%$ & 1.8 & 10.3 & 28.5 & 40.3 & 19.2 \\
Nunca me permito perder el control & $\mathrm{f}$ & 19 & 86 & 223 & 250 & 85 \\
& $\%$ & 2.9 & 13.0 & 33.6 & 37.7 & 12.8 \\
Puedo controlar mis gastos & $\mathrm{f}$ & 7 & 43 & 133 & 264 & 216 \\
& $\%$ & 1.1 & 6.5 & 20.1 & 39.8 & 32.6 \\
Mantengo todo limpio y en orden & $\mathrm{f}$ & 15 & 85 & 182 & 245 & 136 \\
& $\%$ & 2.3 & 12.8 & 27.5 & 37.0 & 20.5 \\
Acostumbro ser disciplinado & $\mathrm{f}$ & 5 & 68 & 177 & 271 & 142 \\
& $\%$ & 0.8 & 10.3 & 26.7 & 40.9 & 21.4 \\
Aun en los momentos más emocionantes puedo mantener el control & $\mathrm{f}$ & 19 & 74 & 169 & 291 & 110 \\
Soy muy bueno guardando secretos & $\%$ & 2.9 & 11.2 & 25.5 & 43.9 & 16.6 \\
& $\mathrm{f}$ & 5 & 11 & 52 & 214 & 381 \\
No pueden desanimarme fácilmente & $\%$ & 0.8 & 1.7 & 7.8 & 32.3 & 57.5 \\
Siempre soy puntual & $\mathrm{f}$ & 42 & 79 & 160 & 224 & 158 \\
Rechazo las cosas que son malas para mí & $\%$ & 6.3 & 11.9 & 24.1 & 33.8 & 23.8 \\
\hline
\end{tabular}

Nota . Totalmente en desacuerdo $=1$, En desacuerdo $=2$, Ni de acuerdo ni en Desacuerdo $=3$, De acuerdo $=4$, Totalmente de Acuerdo $=5$

Fuente: Elaboración propia

Los resultados de la Tabla 4 muestran que, frente a la crisis y sus impredecibles consecuencias, los sujetos se limitan, en lo fundamental, a adquirir productos de primera necesidad, así como productos de limpieza y aseo en general. La alimentación y salud parecieran ser la preocupación fundamental de la población limeña frente a la crisis sanitaria, mientras que en otros aspectos los gastos se contraen significativamente. Esto se explica en la medida de que las familias ya no tienen los mismos niveles de ingresos y además por el peligro de contagio, con los costos que eso supone 
y que excede su capacidad económica. Debido a la importancia que tienen en estas circunstancias, las compras por internet se presentan en una tabla propia (Tabla 5).

\section{Tabla 4}

Comportamiento del consumidor

En el marco de la actual crisis de salud por el coronavirus (COVID-19), ¿qué ha cambiado de las cosas que habitualmente compra?

\begin{tabular}{lcc}
\hline Respuestas & Sí \% & No \% \\
\hline He dejado de comprar productos como vestuario y calzado & 82.7 & 17.3 \\
He dejado de comprar productos asociados a tecnología y electrohogar & 64.6 & 35.4 \\
He dejado de comprar aquellas cosas que no son de primera necesidad & 67.7 & 32.3 \\
He comprado más productos de primera necesidad & 88.7 & 11.3 \\
He dejado de comprar productos más caros para ahorrar & 86.9 & 13.1 \\
He comprado más artículos asociados a limpieza e higiene personal & 86.0 & 14.0 \\
No he cambiado nada & 18.7 & 81.3 \\
He comprado más alimentos no perecibles de los que necesito & 41.9 & 58.1 \\
He comprado más medicamentos y productos asociados a la salud & 61.7 & 38.3 \\
He comprado menos alimentos perecibles & 34.8 & 65.2 \\
\hline
\end{tabular}

Fuente: Elaboración propia

En comparación a las épocas prepandemia, los resultados de la Tabla 5 muestran que la población está cambiando algunos hábitos de consumo, sobre todo en lo que se refiere a las compras online, particularmente en la compra de alimentos y productos tecnológicos. Sin embargo, todavía no es su forma principal de compra, a pesar del tiempo transcurrido desde el inicio de la pandemia.

\section{Tabla 5}

Comportamiento del consumidor

En el entorno actual, marcado por el aislamiento social producto del coronavirus (COVID-19), las compras más recientes que he realizado por internet (comercio online) son:

\begin{tabular}{lcc}
\hline & Sí \% & No \% \\
\hline Realizo compras por internet & 35.1 & 64.9 \\
\hline Alimentos & 39.7 & 60.3 \\
Calzado & 9.7 & 90.3 \\
Vestuario & 20.8 & 79.2 \\
Electrodomésticos & 15.8 & 84.2 \\
Tecnología (computadoras, laptops) & 29.7 & 70.3 \\
Medicamentos & 25.5 & 74.5 \\
Pasajes aéreos y terrestres & 4.7 & 95.3 \\
Alimentos preparados (delivery de restaurantes) & 33.2 & 66.8 \\
\hline
\end{tabular}

Fuente: Elaboración propia 


\section{Análisis Correlacionales}

El análisis de las correlaciones que se presenta en la Tabla 6, muestra que el control emocional se correlaciona de manera significativa y a un nivel medio con la preocupación por la COVID-19 en la muestra estudiada.

Tabla 6

Análisis de Correlación entre el control emocional y la preocupación por la COVID-19

\begin{tabular}{ll}
\hline Variables & Preocupación por la COVID-19 \\
\hline Control emocional & $0.570^{* * *}$ \\
\hline$* \mathrm{p}<.05$ & \\
$\mathrm{~N}=663$ & \\
Fuente: Elaboración propia &
\end{tabular}

El análisis de las correlaciones que se presenta en la Tabla 7, muestra que el control emocional y el comportamiento del consumidor no se correlacionan en la muestra estudiada.

Tabla 7

Análisis de Correlación entre el control emocional y el comportamiento del consumidor

\begin{tabular}{ll}
\hline Variables & Comportamiento del consumidor \\
\hline Control emocional & 0.092 \\
$* \mathrm{p}<.05, \mathrm{~N}=663$ &
\end{tabular}

El análisis de las correlaciones que se presenta en la Tabla 8, muestra que el comportamiento del consumidor y la preocupación por la COVID-19 no se correlacionan en la muestra estudiada.

\section{Tabla 8}

Análisis de Correlación entre el comportamiento del consumidor y la preocupación por la COVID-19

\begin{tabular}{ll}
\hline Variables & Preocupación por la COVID-19 \\
\hline Comportamiento del consumidor & 0.056 \\
\hline$* \mathrm{p}<.05, \mathrm{~N}=663$ &
\end{tabular}

\section{DISCUSIÓN}

El instrumento de recolección de datos fue aplicado durante los primeros quince días de setiembre 2020, siete meses después del inicio del estado de emergencia y las restricciones para contrarrestrar la COVID-19 en el Perú. Los resultados obtenidos nos muestran que la preocupación por la COVID-19 es alta, tal como refleja la Tabla 2, lo que ha llevado a modificar aspectos significativos del comportamiento 
del consumidor, sobre todo, durante los primeros meses de la pandemia. Sin embargo, esta no es lo suficiente fuerte como para modificar más el comportamiento del consumidor y mantenerlo en el tiempo, esto quiere decir que, en la medida de que la pandemia se extendía cada vez más, los consumidores adaptaron sus hábitos de consumo a las restricciones impuestas por el gobierno, como los nuevos horarios de atención que llevó a que se compre en días que no eran los habituales antes de la pandemia y comprar en una sola visita para evitar largos desplazamientos y reducir la exposición al virus (Salas, 2020; El Economista América, 2020). Eso, a su vez, incidió en la compra de proximidad, esto es, en tiendas cercanas a su casa. En esa línea, un estudio reciente de Mastercard Perú (Inga, 2020) muestra que el 85\% de peruanos ha descubierto nuevas tiendas en su barrio.

Según lo observado en la Tabla 3, los hombres y mujeres de la muestra tienden a presentar niveles medios y altos de control emocional, lo cual puede haberlos llevado inicialmente a controlar sus miedos y acatar las medidas impuestas para evitar contagios. Este nivel medio se puede explicar por el tiempo transcurrido desde el inicio de las medidas (15 de marzo de 2020) hasta la fecha de medición de la presente investigación (1-15 de setiembre de 2020). Estudios como el de Torres (2020) muestran que la presencia de la emoción, media entre la racionalidad y una acción de compra, lo que tiende a anular una respuesta racional.

Por otra parte, los niveles de preocupación por la COVID-19, que alcanzan el $57.7 \%$ en el presente trabajo, y que se ven reflejados por la reducción de la movilidad - según estudios realizados por Google, BBVA Research y el Banco Interamericano de Desarrollo (Centro Wiñaq, 2020) - fueron inicialmente altos. No obstante, a medida que las restricciones se alargaban, los habitantes se fueron adaptando a las nuevas circunstancias. Por ejemplo, se ha hecho parte de la rutina el uso de mascarilla, de alcohol en gel o líquido y otros hábitos de limpieza personal y de la casa, el teletrabajo o las clases a distancia en los casos en los que fue posible, etc. Cabe mencionar que el proceso de adaptación a una nueva situación reduce los niveles de preocupación, en este caso por la pandemia. Otro factor que debe ser considerado es la necesidad. Tal como se mencionó en la introducción, la economía peruana es altamente informal, por lo que para el grueso de la población un día no trabajado equivale a un día sin ingresos. Esto llevó a millones de personas a salir a trabajar a las calles, terminando de facto la cuarentena antes de su final oficial a finales de junio (Agencia EFE, 2020). Así, es altamente probable que las personas de la muestra, en uso de sus niveles medios y altos de control emocional, sumado a la gran capacidad de resiliencia que históricamente ha caracterizado a los peruanos, hayan podido adaptarse con éxito a esta situación tan adversa y controlado su preocupación por la pandemia, tomando las medidas sanitarias respectivas para poder continuar, en la medida de lo posible, con sus actividades cotidianas de manera que puedan generar ingresos suficientes para subsistir. 
Según la Tabla 5, un 64.9\% no realiza compras por internet. Esto no supone una novedad dada la cifra del año 2019: solo el 23\% de los peruanos con acceso a internet compraba en línea (Barranzuela, 2019). Factores como la desconfianza en el pago con tarjetas de crédito o la preferencia por ver el producto (Americas Market Intelligence, 2019) explicaban por qué las compras por internet eran aún bajas en el Perú. Sin embargo, las circunstancias de la pandemia forzaron el cambio, principalmente para reducir las salidas (y con ello la exposición al virus). El 35.1\% de la muestra realiza compras por internet, siendo los alimentos preparados (delivery de restaurantes) y tecnología, las categorías donde un mayor porcentaje de la muestra ha realizado compras. La primera categoría, alrededor del $40 \%$, es consistente con lo encontrado por GfK (Ochoa Fattorini, 2020) en el mes de abril de 2020 , cuando ya un $30 \%$ de los encuestados anticipaba que compraría alimentos por internet. Tanto las categorías alimentos preparados (33.2\%) como tecnología (29.7\%) son destacables puesto que no siempre estuvieron abiertas durante el periodo de cuarentena: ambas reabrieron sus operaciones a mediados de mayo. En el caso de la primera, la costumbre de comer fuera fue sustituida por el delivery. Para el caso de la segunda, el porcentaje se explica porque el teletrabajo y la educación a distancia requieren de equipos tecnológicos y/o accesorios (como cámaras web, monitores, audífonos, etc.) que permitan una conexión fluida y estable, por lo que podemos considerar estas compras como inversiones.

En lo que se refiere a los análisis de correlación, la tabla 6, nos informa de la existencia de una correlación significativa entre el control emocional y la preocupación por la COVID-19 en un nivel medio. Este nivel medio de correlación se puede explicar por el tiempo transcurrido, es decir que después de seis meses del inicio de las medidas restrictivas, la población ha debido adecuarse a las nuevas condiciones y salir a trabajar o atender situaciones familiares (Torres, 2020). La delicada situación económica de la mayoría de la población los obliga a desafiar el peligro del contagio y salir a buscar el sustento diario de si mismo y de sus familias.

Así mismo, la Tabla 7 muestra que no existe correlación entre el control emocional y el comportamiento del consumidor, probablemente porque la situación excepcional de la pandemia, a pesar del miedo inicial, no alteró significativamente los hábitos de consumo del grueso de la población, quienes siguieron dirigiendo su atención inmediata a la satisfacción de sus necesidades básicas. En poblaciones con alto porcentaje de informalidad como la peruana, el vivir para el día a día hace que el consumo sea para satisfacer necesidades inmediatas, como la alimentación (Sánchez y Chafloque, 2019). Esto lo confirma lo señalado en la Tabla 4: un 67.7\% ha dejado de comprar productos que no son de primera necesidad, y un $86.9 \%$ ya no compra productos caros o productos de marca, que regularmente tienen mayor precio que los de las marcas propias de los comercios, conocidos como marcas blancas. 
La Tabla 8 muestra que no existe una correlación entre el comportamiento del consumidor y la preocupación por la COVID-19. Ciertamente la preocupación por la pandemia influyó en el comportamiento del consumidor en el inicio de la emergencia (El Comercio, 2020), cuando las personas, sobre todo aquellas con capacidad de compra, salieron desesperadamente a comprar todo lo que pudieron ante el temor de desabastecimientos, incluso cuando el gobierno garantizó el suministro normal de productos de primera necesidad, pero progresivamente se moderó. Se debe insistir en el hecho de que la mayoría de la población peruana no se puede permitir desbordarse en la compra, pues no tiene como hacerlo; no solo por un tema de ingresos, sino de capacidad de almacenamiento. Por ejemplo, según el INEI (Perú 21, 2020), el 47.5\% de las familias a nivel nacional no tenía un refrigerador en 2019, siendo esto mayor en los sectores D y E.

Es verdad que por su naturaleza el alcance de esta investigación corresponde a la ciudad de Lima, sin embargo, por la importancia de los mismos, se debe considerar como punto de referencia para otras investigaciones similares o para la toma de decisiones respecto del tratamiento que se le debe dar a la pandemia en los planos económicos, sociales y sobre todo en los aspectos emocionales.

En síntesis, se puede concluir que, los cambios en el consumidor estuvieron reflejados por las restricciones impuestas: se buscó proximidad, hacer compras en un solo turno para reducir las salidas, se dejó de consumir porque los comercios no estaban abiertos o por una reducción de ingresos. Si bien la preocupación pudo tener un rol, este no fue lo suficientemente grande como para tener un impacto significativo sobre qué comprar o el modo de hacerlo. Por otra parte, los resultados muestran que, frente a la crisis y sus impredecibles consecuencias, los sujetos se limitan, en lo fundamental, a adquirir productos de primera necesidad, así como también productos de limpieza y aseo en general. Alimentación y salud parecieran ser la preocupación fundamental de la población limeña frente a la crisis sanitaria, mientras que en otros aspectos los gastos se contraen significativamente. Esto se explica en la medida de que las familias ya no tienen los mismos niveles de ingresos, además el peligro de contagio, con los costos que eso supone y que excede su capacidad económica.

Finalmente se puede mencionar que, tras la estabilización de la curva de contagio, diversos medios y especialistas han mencionado la posibilidad de una segunda ola en el Perú, tomando como base lo ocurrido en Europa y Estados Unidos. Si bien está próxima, la puesta a disposición de las primeras vacunas contra el coronavirus, mientras no se inmunice a buena parte de la población, la segunda ola seguirá siendo una posibilidad. En los países donde esto ya es una realidad, como Alemania, España o Estados Unidos, se ha observado que el solo temor a una potencial cuarentena (total o parcial) dispara las compras compulsivas, tal cual ocurrió en marzo (Löhr, Schwenn, \& Diemand, 2020; Riley Moffat, 2020). Por ello, podríamos esperar un 
comportamiento similar en países como Perú o Chile, que, a inicios de diciembre de 2020, han tenido éxito controlando sus contagios, si es que esta segunda ola eventualmente ocurre. Así, las empresas productoras y los comercios podrán anticipar una demanda extraordinaria y producir y distribuir los bienes en concordancia a ello. Solo generar la sensación de que no habrá escasez de esos bienes de primera necesidad puede tener un buen impacto en la población, que percibirá que, pese a la situación, puede tener la situación bajo cierto grado de control, lo suficiente como para no desencadenar el mismo escenario de los primeros meses de la pandemia.

\section{Agradecimientos}

Los autores quieren expresar su profundo agradecimiento a la señorita Shirley Puente Gonzales por su generosa ayuda en la realización de la presente investigación, así como también a los estudiantes del postgrado de la Universidad Nacional de Educación (UNE) y la Universidad Peruana Cayetano Heredia (UPCH) por su destacada colaboración.

\section{Fuentes de financiamiento}

La investigación fue financiada con recursos de los propios autores.

\section{Aspectos éticos}

En la presente investigación se pidió el consentimiento de las personas participantes para desarrollar el cuestionario. Además, se aseguró la confidencialidad y privacidad de los datos obtenidos, los mismos que quedaran bajo el cuidado del equipo de investigación. En todos los casos se deja constancia de que la participación fue totalmente voluntaria.

\section{Conflicto de intereses}

Los autores declaran no tener ningún conflicto de interés económico, institucional, laboral o personal al realizar el manuscrito.

\section{REFERENCIAS}

Agencia EFE. (28 de mayo de 2020). El Perú informal no espera al final de la cuarentena para trabajar. Obtenido de EFE: https://www.efe.com/efe/america/economia/elperu-informal-no-espera-al-final-de-la-cuarentena-para-trabajar/20000011-4257682

Americas Market Intelligence. (15 de diciembre de 2019). Lo que más compran los peruanos en internet. Obtenido de Americas Market Intelligence: https://americasmi.com/ insights/lo-que-mas-compran-los-peruanos-en-internet/

Ariely, D. (2010). Predictably Irrational. New York: HarperCollins. 
Barranzuela, I. (noviembre de 2019). El 23\% de los peruanos compra por internet, según la CCL. Obtenido de Mercado Negro: https://www.mercadonegro.pe/retail/el-23-delos-peruanos-compra-por-internet-segun-la-ccl/

Bécares, R. (10 de marzo de 2020). "Con el coronavirus es una locura, estamos sacando toda la comida del almacén, pero no sabemos cuánto durará". Obtenido de El Mundo: https:/www.elmundo.es/madrid/2020/03/10/5e677cblfdddff4cbb8b45c4.html

Centro Wiñaq. (2 de octubre de 2020). ¿Fuimos los peruanos irresponsables en el cumplimiento de la cuarentena? . Obtenido de RPP Noticias: https://rpp.pe/columnistas/centrowinaq/fuimos-los-peruanos-irresponsables-en-el-cumplimiento-de-lacuarentena-noticia-1296129

Corkery, M., \& Maheshwari, S. (13 de marzo de 2020). Is There Really a Toilet Paper Shortage? Obtenido de The New York Times: https://www.nytimes.com/2020/03/13/ business/toilet-paper-shortage.html

Chóliz, M. (2005). Psicología de la emoción: el proceso emocional. Universidad de Valencia. (citado el 01 de diciembre de 2020). Disponible en: http://www.uv.es/=choliz/ Proceso\%20emocional.pdf

De Leersnyder, J., Boiger, M. \& Mesquita, B. (2013). Cultural regulation of emotion: individual, relational, and structural sources. Frontiers in Psychology, 2013; 55(4), 1-11. https://doi.org/10.3389/fpsyg.2013.00055

Del Pozo, J. A. (2013). Técnicas de comunicación oral y grupal. Alicante: Editorial Club Universitario.

Díaz, N. (25 de marzo de 2020). Registran largas filas en supermercados tras anuncio de cuarentena para 7 comunas de la RM . Obtenido de Bio Bio Chile: https://www.biobiochile.cl/noticias/nacional/region-metropolitana/2020/03/25/registran-largas-filas-en-supermercados-tras-anuncio-de-cuarentena-para-7-comunas-de-la-rm.shtml

El Comercio. (12 de marzo de 2020). Coronavirus en el Perú: largas colas y anaqueles vacios en principales supermercados de Lima y Callao . Obtenido de El Comercio: https:/elcomercio.pe/lima/sucesos/coronavirus-en-peru-largas-colas-y-anaqueles-vacios-en-principales-supermercados-de-lima-y-callao-makro-tottus-covid19-noticia/?ref=ecr

El Economista América. (6 de mayo de 2020). Este es el perfil de consumo de los peruanos en la cuarentena. Obtenido de El Economista América: https:/www.eleconomistaamerica.pe/economia-eAm-peru/noticias/10526525/05/20/Este-es-el-perfil-deconsumo-de-los-peruanos-en-la-cuarentena.html

El Peruano. (13 de enero de 2021). Ministra de Salud: "Estamos en una segunda ola". Obtenido de El Peruano: https://elperuano.pe/noticia/113365-ministra-de-salud-estamos-en-una-segunda-ola

Gamero, J., \& Pérez, J. (setiembre de 2020). Perú: Impacto de la COVID-19 en el empleo y los ingresos laborales. Obtenido de Organización Internacional del Trabajo: https:// www.ilo.org/wcmsp5/groups/public/---americas/---ro-lima/documents/publication/ wcms_756474.pdf 
Garbe, L., Rau, R., \& Toppe, T. (2020). Influence of perceived threat of Covid-19 and HEXACO personality traits on toilet paper stockpiling. PLOS ONE, 15(6), e0234232. https://doi.org/10.1371/journal.pone.0234232

Goleman, D. (26 de julio de 2015). Daniel Goleman: Self-Regulation: A Star Leader's Secret Weapon. Obtenido de Daniel Goleman: http://www.danielgoleman.info/danielgoleman-self-regulation-a-star- leaders-secret-weapon/

Grace, R., \& Sakkal, P. (5 de marzo de 2020). What role did rumour play in Australia's toilet paper frenzy? Obtenido de The Sydney Morning Herald: https://www. smh.com.au/national/what-role-did-rumour-play-in-australia-s-toilet-paper-frenzy20200304-p546tt.html

Gross, J. (2009). Handbook of Emotion Regulation. New York: Guilford Press

Grolnick, W. S., McMenamy, J. M., \& Kurowski, C. O. (2006). Emotional self-regulation in infancy and toddlerhood. En L. Balter \& C. S. Tamis-LeMonda (Eds.), Child psychology: A handbook of contemporary issues (pp. 3-25). Nueva York, NY: Psychology Press.

Inga, C. (25 de noviembre de 2020). Los peruanos han vuelto a mirar a los negocios del barrio por la pandemia, ¿a cuáles y por qué? Obtenido de El Comercio: https:// elcomercio.pe/economia/dia-1/negocios-los-peruanos-han-vuelto-a-mirar-a-los-negocios-del-barrio-por-la-pandemia-que-razones-lo-explican-noticia/?ref=ecr

Kahneman, D. (2011). Pensar rápido, pensar despacio. Barcelona: Debate.

La Nación. (11 de setiembre de 2020). Coronavirus hoy en Perú: cuántos casos se registran al 10 de Septiembre. Obtenido de Yahoo Finanzas: https:/es.finance.yahoo.com/ noticias/coronavirus-perú-cuántos-casos-registran-041300686.html

La República. (20 de diciembre de 2020). Perú es noticia en el mundo por aglomeraciones en compras navideñas. Obtenido de La República: https://larepublica.pe/ mundo/2020/12/20/peru-es-noticia-en-el-mundo-por-aglomeraciones-en-comprasnavidenas/?ref=lre

Löhr, J., Schwenn, K., \& Diemand, S. (18 de octubre de 2020). Anstieg der Corona-Infektionen: Die Deutschen hamstern wieder. Obtenido de Frankfurter Allgemeine Zeitung: https://www.faz.net/aktuell/wirtschaft/corona-krise-die-deutschen-hamsternwieder-17007920.html

Mullainathan, S., \& Shafir, E. (2014). Scarcity: The True Cost of Not Having Enough: Why having too little means so much. Londres: Penguin.

Ochoa, V. (16 de abril de 2020). El 58\% de los consumidores online elevan sus compras en alimentos y medicamentos. Obtenido de Gestión: https://gestion.pe/tendencias/ el-58-de-los-consumidores-online-elevan-sus-compras-en-alimentos-y-medicamentos-noticia/?ref=gesr

Organización Mundial de la Salud (OMS). (11 de marzo de 2020). Alocución de apertura del Director General de la OMS en la rueda de prensa sobre la COVID-19 celebrada el 11 de marzo de 2020. Obtenido de Organización Mundial de la Salud: https:// 
www.who.int/es/dg/speeches/detail/who-director-general-s-opening-remarks-at-themedia-briefing-on-covid-19---11-march-2020

Perú 21. (11 de abril de 2020). Coronavirus en Perú: Problemas para congelar alimentos en hogares habrían contribuido a aglomeraciones . Obtenido de Perú 21: https:// peru21.pe/economia/problemas-para-congelar-alimentos-en-hogares-habrian-contribuido-a-aglomeraciones-noticia/?ref=p21r

Piqueras, J., Ramos, V., Martínez, A. \& Oblitas, L. (2009). Emociones negativas y su impacto en la salud mental y física. Suma Psicológica; 2009;16(2), 85-112. Disponible en: https://www.redalyc.org/articulo.oa?id=134213131007

Presidencia del Consejo de Ministros (PCM). (15 de marzo de 2020). Decreto Supremo que declara Estado de Emergencia Nacional por las graves circunstancias que afectan la vida de la Nación a consecuencia del brote del COVID-19 (DS N442020-PCM). Obtenido de El Peruano: https://busquedas.elperuano.pe/normaslegales/ decreto-supremo-que-declara-estado-de-emergencia-nacional-po-decreto-supremon-044-2020-pcm-1864948-2/

Riley, A. (21 de noviembre de 2020). No Bacon, No Bath Tissue: Panic Buying Returns on Covid Jump. Obtenido de Bloomberg: https://www.bloomberg.com/news/articles/2020-11-21/no-bacon-no-bath-tissue-panic-buying-s-back-with-covid-surge

RPP. (11 de marzo de 2020). Coronavirus: Compras masivas harían que los precios suban innecesariamente. Obtenido de RPP Noticias: https://rpp.pe/economia/economia/ coronavirus-compras-masivas-harian-que-los-precios-suban-innecesariamente-noticia-1250945

Salas, L. (3 de diciembre de 2020). Real Plaza: "Para noviembre y diciembre, las ventas estarán entre un 15\% y 20\% por debajo del 2019”. Obtenido de El Comercio: https:// elcomercio.pe/economia/negocios/real-plaza-hoy-la-gente-es-mas-transaccional-laconversion-es-mas-alta-y-el-ticket-promedio-tambien-es-mayor-entrevista-centrocomercial-grupo-intercorp-puruchuco-centro-civico-ncze-noticia/?ref=ecr

Salazar, E. (21 de mayo de 2020). Guía práctica para el Estado de Emergencia y la cuarentena por el Covid-19 . Obtenido de Ojo Público: https://ojo-publico.com/1669/ covid-19-guia-practica-para-el-estado-de-emergencia-y-la-cuarentena

Sánchez, M, y Chafloque, R. (2019). La informalidad laboral en el Perú: Un mapa nacional basado en ENAHO. Lima. Fondo Editorial USMP.

Stalinski, S. (3 de marzo de 2020). Sinn und Unsinn von "Hamsterkäufen". Obtenido de Taggeschau: https://www.tagesschau.de/inland/corona-hamsterkaufe-101.html

Taylor, S. (2019). The Psychology of Pandemics: Preparing for the Next Global Outbreak of Infectious Disease. Cambridge: Cambridge Scholars Publishing.

Torres, M. (2020). The Influence of Economic Literacy and Emotional Control on Credit Card Consumption among Master Students in Lima, Peru [Tesis de Maestría]. Universidad Técnica de Múnich, Alemania. 
TVPerú. (15 de junio de 2020). Presidente Martín Vizcarra: "Ahora nuestro lema es Primero mi salud" . Obtenido de TVPerú: https://www.tvperu.gob.pe/noticias/nacionales/ presidente-martin-vizcarra-ahora-nuestro-lema-es-primero-mi-salud

Van Bavel, J. J.; Baicker, K.; Boggio, P. S.; Capraro, V.; Cichocka, A.; Cikara, M.; ...; Willer, R. (2020). Using social and behavioural science to support COVID-19 pandemic response. Nature Human Behaviour, 1-12. 4, 460-471. doi: 10.31234/osf.io/y38m9

Worldometer. (7 de diciembre de 2020). Coronavirus cases: Peru. Obtenido de Worldometer: https://www.worldometers.info/coronavirus/country/peru/ 